\title{
HYPERSPHERES IN EUCLIDEAN AND MINKOWSKI 4-SPACES AS ALMOST PARACONTACT ALMOST PARACOMPLEX RIEMANNIAN MANIFOLDS
}

\author{
MANCHO MANEV AND VESELINA TAVKOVA
}

\begin{abstract}
Almost paracontact almost paracomplex Riemannian manifolds of the lowest dimension are studied. Such structures are constructed on hyperspheres in 4-dimensional spaces, Euclidean and pseudo-Euclidean, respectively. The obtained manifolds are studied and characterised in terms of the classification used and their geometric properties.
\end{abstract}

\section{Introduction}

In 12, I. Sato introduced the notion of almost paracontact Riemannian structure on a differentiable manifold of arbitrary dimension so that this structure is compatible with a Riemannian metric such that the metric preserves the structure endomorphism on the paracontact distribution. Later, other geometers (K. Matsumoto, T. Adati, T. Miyazawa, S. Sasaki) joined Sato in the initial development of the differential geometry of almost paracontact Riemannian manifolds (e.g. 11, 11]).

Another type of structure-metric compatibility is known in addition to the above. If the structure endomorphism induces an anti-isometry with respect to the metric on the paracontact distribution of each tangent fibre, then it is said that the manifold has an almost paracontact metric structure (see, e.g., [3, 13]).

The restriction of the almost paracontact structure on the paracontact distribution is an almost product structure. In 10, A.M. Naveira gives a classification of Riemannian almost product manifolds with respect to the covariant derivative of the almost product structure regarding the Levi-Civita connection of the Riemannian metric.

Almost paracontact Riemannian manifolds of Sasaki type $(n, n)$ are classified in [6]. For them, the induced almost product structure on the paracontact distribution is traceless and it is called an almost paracomplex structure (4). These manifolds are necessarily odd-dimensional and are called almost paracontact almost paracomplex Riemannian manifolds in [7.

An object of particular interest in our research is the case of the lowest dimension (which is three) of almost paracontact almost paracomplex Riemannian manifolds. In this regard, we study their properties in $[7,8,9$.

In the present work, we use two different approaches to construct an almost paracontact almost paracomplex Riemannian manifold on a hypersphere. The first case is of a hypersphere in Euclidean space $\mathbb{E}^{4}$ and the second is of a time-like

2010 Mathematics Subject Classification. Primary 53C15, 53C45; Secondary 53D15.

Key words and phrases. Almost paracontact structure, almost paracomplex structure, Riemannian metric, hypersphere, space form, constant sectional curvature. 
hypersphere in pseudo-Euclidean space $\mathbb{E}_{1}^{4}$ (i.e. Minkowski space). Similar research is made for almost contact B-metric hyperspheres in [2, 5].

The purpose of this paper is to study the basic geometric characteristics of the considered manifolds. The obtained results will provide explicit examples of the lowest dimension of the manifolds under study and will contribute to the understanding of their geometry.

The paper is organized as follows. In Sect. 2, we recall some necessary basic definitions and properties for the studied manifolds. In Sect. 3 and Sect. 4, we construct and characterize such manifolds on hyperspheres in $\mathbb{E}^{4}$ and $\mathbb{E}_{1}^{4}$, respectively.

\section{Almost paracontact almost paracomplex Riemannian manifolds}

Let us consider an almost paracontact almost paracomplex Riemannian manifold $(\mathcal{M}, \phi, \xi, \eta, g)$, i.e. $\mathcal{M}$ is a real differentiable manifold of dimension $(2 n+1)$ equipped with an almost paracontact almost paracomplex structure $(\phi, \xi, \eta)$ and a Riemannian metric $g$. Namely, $\phi$ is a tensor field of type $(1,1)$ (known as a paracontact endomorphism) of the tangent bundle $T \mathcal{M}$ of $\mathcal{M}, \xi$ is a Reeb vector field and $\eta$ is its dual 1-form, which together with $g$ satisfy the following conditions: [12], 6

$$
\begin{gathered}
\phi^{2}=\mathcal{I}-\eta \otimes \xi, \quad \eta(\xi)=1, \quad \eta \circ \phi=0, \quad \phi \xi=0, \quad \operatorname{tr} \phi=0, \\
g(\phi x, \phi y)=g(x, y)-\eta(x) \eta(y),
\end{gathered}
$$

where $\mathcal{I}$ denotes the identity on $T \mathcal{M}$.

Here and further $x, y, z, w$ will stand for arbitrary elements of the Lie algebra $\mathfrak{X}(\mathcal{M})$ of tangent vector fields on $\mathcal{M}$ or vectors in the tangent space $T_{p} \mathcal{M}$ at $p \in \mathcal{M}$.

Let us denote the Levi-Civita connection of $g$ by $\nabla$. The fundamental tensor $F$ of type $(0,3)$ on $(\mathcal{M}, \phi, \xi, \eta, g)$ is defined by

$$
F(x, y, z)=g\left(\left(\nabla_{x} \phi\right) y, z\right) .
$$

It has the following basic properties with respect to the structure

$$
\begin{aligned}
F(x, y, z) & =F(x, z, y) \\
& =-F(x, \phi y, \phi z)+\eta(y) F(x, \xi, z)+\eta(z) F(x, y, \xi) .
\end{aligned}
$$

The relations of $\nabla \xi$ and $\nabla \eta$ with $F$ are as follows:

$$
\left(\nabla_{x} \eta\right)(y)=g\left(\nabla_{x} \xi, y\right)=-F(x, \phi y, \xi) .
$$

Let $\left\{\xi ; e_{i}\right\}(i=1,2, \ldots, 2 n)$ be a basis of $T_{p} \mathcal{M}$ at an arbitrary point $p \in \mathcal{M}$ and $g^{i j}$ are the components of the inverse matrix of $g$. Using this basis, the structure $(\phi, \xi, \eta)$ and the metric $g$, the following 1 -forms (known as Lee forms) are associated with $F$ :

$$
\theta(z)=g^{i j} F\left(e_{i}, e_{j}, z\right), \quad \theta^{*}(z)=g^{i j} F\left(e_{i}, \phi e_{j}, z\right), \quad \omega(z)=F(\xi, \xi, z) .
$$

In 6], a classification of almost paracontact almost paracomplex Riemannian manifolds is made with respect to basic properties of $F$ with respect to the tensor structure of the studied manifold. This classification consists of 11 basic classes $\mathcal{F}_{1}, \mathcal{F}_{2}, \ldots, \mathcal{F}_{11}$. Furthermore, the components $F^{s}(s \in\{1,2, \ldots, 11\})$ of $F$, which correspond to the classes $\mathcal{F}_{s}$, are determined in [7]. The latter approach provides an alternative way to determine the basic classes of the considered classification. Namely, the manifold $(\mathcal{M}, \phi, \xi, \eta, g)$ belongs to $\mathcal{F}_{s}$ if and only if the equality $F=F^{s}$ is valid. As a corollary we have the following. A manifold of the studied type belongs 
to a direct sum of two or more basic classes, i.e. $(\mathcal{M}, \phi, \xi, \eta, g) \in \mathcal{F}_{i} \oplus \mathcal{F}_{j} \oplus \cdots$, if and only if the tensor $F$ on $(\mathcal{M}, \phi, \xi, \eta, g)$ is the sum of the corresponding components $F^{i}, F^{j}, \ldots$ of $F$, i.e. the following condition is satisfied $F=F^{i}+F^{j}+\cdots$.

Let $(\mathcal{M}, \phi, \xi, \eta, g)$ have the lowest dimension (i.e. $\operatorname{dim} \mathcal{M}=3)$ and let the set of vectors $\left\{e_{0}, e_{1}, e_{2}\right\}$ be a $\phi$-basis of $T_{p} \mathcal{M}$ which satisfies the following conditions:

$$
\begin{gathered}
\phi e_{0}=0, \quad \phi e_{1}=e_{2}, \quad \phi e_{2}=e_{1}, \quad \xi=e_{0}, \\
\eta\left(e_{0}\right)=1, \quad \eta\left(e_{1}\right)=\eta\left(e_{2}\right)=0, \\
g\left(e_{i}, e_{j}\right)=\delta_{i j}, \quad i, j \in\{0,1,2\} .
\end{gathered}
$$

According to [7], the components $F_{i j k}=F\left(e_{i}, e_{j}, e_{k}\right), \theta_{k}=\theta\left(e_{k}\right), \theta_{k}^{*}=\theta^{*}\left(e_{k}\right)$ and $\omega_{k}=\omega\left(e_{k}\right)$ of $F, \theta, \theta^{*}$ and $\omega$, respectively, with respect to the $\phi$-basis $\left\{e_{0}, e_{1}, e_{2}\right\}$ are determined as follows:

$$
\begin{gathered}
\theta_{0}=F_{110}+F_{220}, \quad \theta_{1}=F_{111}=-F_{122}=-\theta_{2}^{*}, \\
\theta_{0}^{*}=F_{120}+F_{210}, \quad \theta_{2}=F_{222}=-F_{211}=-\theta_{1}^{*}, \\
\omega_{0}=0, \quad \omega_{1}=F_{001}, \quad \omega_{2}=F_{002} .
\end{gathered}
$$

Hence, the components $F^{s}, s \in\{1,2, \ldots, 11\}$, of $F$ on $(\mathcal{M}, \phi, \xi, \eta, g)$ in the corresponding basic classes $\mathcal{F}_{s}$ have the following form: [7]

$$
\begin{aligned}
F^{1}(x, y, z) & =\left(x^{1} \theta_{1}-x^{2} \theta_{2}\right)\left(y^{1} z^{1}-y^{2} z^{2}\right) \\
F^{2}(x, y, z) & =F^{3}(x, y, z)=0 \\
F^{4}(x, y, z) & =\frac{\theta_{0}}{2}\left\{x^{1}\left(y^{0} z^{1}+y^{1} z^{0}\right)+x^{2}\left(y^{0} z^{2}+y^{2} z^{0}\right)\right\} \\
F^{5}(x, y, z) & =\frac{\theta_{0}^{*}}{2}\left\{x^{1}\left(y^{0} z^{2}+y^{2} z^{0}\right)+x^{2}\left(y^{0} z^{1}+y^{1} z^{0}\right)\right\} \\
F^{6}(x, y, z) & =F^{7}(x, y, z)=0 \\
F^{8}(x, y, z) & =\lambda\left\{x^{1}\left(y^{0} z^{1}+y^{1} z^{0}\right)-x^{2}\left(y^{0} z^{2}+y^{2} z^{0}\right)\right\} \\
\lambda & =F_{110}=-F_{220} \\
F^{9}(x, y, z) & =\mu\left\{x^{1}\left(y^{0} z^{2}+y^{2} z^{0}\right)-x^{2}\left(y^{0} z^{1}+y^{1} z^{0}\right)\right\} \\
\mu & =F_{120}=-F_{210} ; \\
F^{10}(x, y, z) & =\nu x^{0}\left(y^{1} z^{1}-y^{2} z^{2}\right), \quad \nu=F_{011}=-F_{022} ; \\
F_{11}(x, y, z) & =x^{0}\left\{\omega_{1}\left(y^{0} z^{1}+y^{1} z^{0}\right)+\omega_{2}\left(y^{0} z^{2}+y^{2} z^{0}\right)\right\}
\end{aligned}
$$

where the decompositions $x=x^{i} e_{i}, y=y^{i} e_{i}, z=z^{i} e_{i}$ with respect to $\left\{e_{0}, e_{1}, e_{2}\right\}$ are used.

By virtue of (2.4), it is determined in 77 that the studied 3-dimensional manifolds can belong only to the basic classes $\mathcal{F}_{1}, \mathcal{F}_{4}, \mathcal{F}_{5}, \mathcal{F}_{8}, \mathcal{F}_{9}, \mathcal{F}_{10}, \mathcal{F}_{11}$ and their direct sums.

The Nijenhuis tensor $N$ of the structure $(\phi, \xi, \eta)$ is defined by the equality $N(x, y)=[\phi, \phi](x, y)-\mathrm{d} \eta(x, y) \xi$, where the Nijenhuis torsion of $\phi$ is determined by $[\phi, \phi](x, y)=[\phi x, \phi y]+\phi^{2}[x, y]-\phi[\phi x, y]-\phi[x, \phi y]$ and $\mathrm{d} \eta$ is the exterior derivative of $\eta$ given by $\mathrm{d} \eta(x, y)=\left(\nabla_{x} \eta\right) y-\left(\nabla_{y} \eta\right) x$. The corresponding tensor of type $(0,3)$ of the Nijenhuis tensor on $(\mathcal{M}, \phi, \xi, \eta, g)$ is defined by the equality $N(x, y, z)=g(N(x, y), z)$. According to [7, we express $N$ in terms of $F$ as follows:

$$
\begin{aligned}
N(x, y, z)= & F(\phi x, y, z)-F(\phi y, x, z)-F(x, y, \phi z)+F(y, x, \phi z) \\
& +\eta(z)\{F(x, \phi y, \xi)-F(y, \phi x, \xi)\} .
\end{aligned}
$$


The associated Nijenhuis tensor $\widehat{N}$ of the structure $(\phi, \xi, \eta, g)$ is defined by the following way $\widehat{N}(x, y)=\{\phi, \phi\}(x, y)-\left(\mathfrak{L}_{\xi} g\right)(x, y) \xi$. In the latter equality, $\{\phi, \phi\}$ is the symmetric tensor of type $(1,2)$ determined by $\{\phi, \phi\}(x, y)=\{\phi x, \phi y\}+$ $\phi^{2}\{x, y\}-\phi\{\phi x, y\}-\phi\{x, \phi y\}$ for $\{x, y\}=\nabla_{x} y+\nabla_{y} x$ and $\mathfrak{L}_{\xi} g$ is the Lie derivative of $g$ along $\xi$ expressed by $\left(\mathfrak{L}_{\xi} g\right)(x, y)=\left(\nabla_{x} \eta\right) y+\left(\nabla_{y} \eta\right) x$. The corresponding tensor of type $(0,3)$ of the associated Nijenhuis tensor is defined by $\widehat{N}(x, y, z)=$ $g(\widehat{N}(x, y), z)$. In [7], we express $\widehat{N}$ by $F$ as follows:

$$
\begin{aligned}
\widehat{N}(x, y, z)= & F(\phi x, y, z)+F(\phi y, x, z)-F(x, y, \phi z)-F(y, x, \phi z) \\
& +\eta(z)\{F(x, \phi y, \xi)+F(y, \phi x, \xi)\} .
\end{aligned}
$$

The curvature tensor $R$ of type $(1,3)$ for $\nabla$ is defined as usually by $R=[\nabla, \nabla]-$ $\nabla_{[,]}$. The corresponding $(0,4)$-tensor is denoted by the same letter and it is given by $R(x, y, z, w)=g(R(x, y) z, w)$.

The Ricci tensor $\rho$ and the scalar curvature $\tau$ for $R$ as well as their associated quantities are determined respectively by:

$$
\begin{array}{ll}
\rho(y, z)=g^{i j} R\left(e_{i}, y, z, e_{j}\right), & \tau=g^{i j} \rho\left(e_{i}, e_{j}\right), \\
\rho^{*}(y, z)=g^{i j} R\left(e_{i}, y, z, \phi e_{j}\right), & \tau^{*}=g^{i j} \rho^{*}\left(e_{i}, e_{j}\right) .
\end{array}
$$

Moreover, we use the Kulkarni-Nomizu product $g \otimes h$ of two $(0,2)$-tensors $g$ and $h$ defined by

$$
\begin{aligned}
(g \oplus h)(x, y, z, w) & =g(x, z) h(y, w)-g(y, z) h(x, w) \\
& +g(y, w) h(x, z)-g(x, w) h(y, z) .
\end{aligned}
$$

Obviously, $g \bowtie h$ has the basic properties of $R$ if and only if $g$ and $h$ are symmetric.

Let $\alpha$ be a non-degenerate 2-plane in $T_{p} \mathcal{M}, p \in \mathcal{M}$, having a basis $\{x, y\}$. The sectional curvature $k(\alpha ; p)$ is determined by

$$
k(\alpha ; p)=-\frac{2 R(x, y, y, x)}{(g \otimes g)(x, y, y, x)} .
$$

\section{A hypersphere with the studied structure in Euclidean 4-space}

Let $\mathbb{E}^{4}$ be the Euclidean space $\left(\mathbb{R}^{4},\langle\cdot, \cdot\rangle\right)$, where $\langle\cdot, \cdot\rangle$ is the usual Euclidean inner product determined by

$$
\langle x, y\rangle=x^{1} y^{1}+x^{2} y^{2}+x^{3} y^{3}+x^{4} y^{4}
$$

for $x\left(x^{1}, x^{2}, x^{3}, x^{4}\right), y\left(y^{1}, y^{2}, y^{3}, y^{4}\right)$ from $\mathbb{R}^{4}$.

Then, we consider a hypersphere $S_{1}$ in $\mathbb{E}^{4}$ at the origin with a real radius $r$ identifying an arbitrary point $p$ in $\mathbb{E}^{4}$ with its position vector $z$, i.e.

$$
S_{1}: \quad\langle z, z\rangle=r^{2} .
$$

It has the following parametrization

$$
z\left(r \cos u^{1} \cos u^{2}, r \cos u^{1} \sin u^{2}, r \sin u^{1} \cos u^{0}, r \sin u^{1} \sin u^{0}\right),
$$

where $u^{0}, u^{1}, u^{2}$ are real parameters such as $u^{0}, u^{1}, u^{2} \in[0 ; 2 \pi), u^{1} \neq \frac{k \pi}{2}$ for $k \in$ $\{0,1,2,3\}$. Consequently, the local basic vectors $\partial_{i}=\frac{\partial z}{\partial u^{i}}, i \in\{0,1,2\}$ have the following inner products:

$$
\begin{aligned}
& \left\langle\partial_{0}, \partial_{0}\right\rangle=r^{2} \sin ^{2} u^{1}, \quad\left\langle\partial_{1}, \partial_{1}\right\rangle=r^{2}, \quad\left\langle\partial_{2}, \partial_{2}\right\rangle=r^{2} \cos ^{2} u^{1}, \\
& \left\langle\partial_{i}, \partial_{j}\right\rangle=0, i \neq j .
\end{aligned}
$$


Substituting $e_{i}=\frac{1}{\sqrt{\left\langle\partial_{i}, \partial_{i}\right\rangle}} \partial_{i}, i \in\{0,1,2\}$, we obtain an orthonormal basis $\left\{e_{i}\right\}$, $i \in\{0,1,2\}$ as follows

$$
e_{0}=\frac{\varepsilon_{2}}{r \sin u^{1}} \partial_{0}, \quad e_{1}=\frac{1}{r} \partial_{1}, \quad e_{2}=\frac{\varepsilon_{1}}{r \cos u^{1}} \partial_{2},
$$

where $\varepsilon_{1}=\operatorname{sgn}\left(\cos u^{1}\right), \varepsilon_{2}=\operatorname{sgn}\left(\sin u^{1}\right)$.

Next, we introduce an almost paracontact almost paracomplex structure $(\phi, \xi, \eta)$ on $S_{1}$ determined as shown in (2.2). The metric $g$ on the hypersurface is the restriction of $\langle\cdot, \cdot\rangle$ on $S_{1}$. Therefore, $\left\{e_{i}\right\}, i \in\{0,1,2\}$ is an orthonormal $\phi$-basis with respect to $g$ on $T_{p} S_{1}$ at $p \in S_{1}$, i.e. (2.3) is satisfied. Thus, we obtain for $\left(S_{1}, \phi, \xi, \eta, g\right)$ the following

Proposition 3.1. The manifold $\left(S_{1}, \phi, \xi, \eta, g\right)$ is a 3-dimensional almost paracontact almost paracomplex Riemannian manifold.

Using (3.2), we calculate the following commutators of the basic vectors $e_{i}$ :

$$
\left[e_{0}, e_{1}\right]=\frac{1}{r} \cot u^{1} e_{0}, \quad\left[e_{0}, e_{2}\right]=0, \quad\left[e_{1}, e_{2}\right]=\frac{1}{r} \tan u^{1} e_{2} .
$$

According to the latter equations and the Koszul equality for $\nabla$ of $g$, i.e.

$$
2 g\left(\nabla_{E_{i}} E_{j}, E_{k}\right)=g\left(\left[E_{i}, E_{j}\right], E_{k}\right)+g\left(\left[E_{k}, E_{i}\right], E_{j}\right)+g\left(\left[E_{k}, E_{j}\right], E_{i}\right),
$$

we obtain the components of the covariant derivatives of $e_{i}$ with respect to $\nabla$ :

$$
\begin{array}{ll}
\nabla_{e_{0}} e_{0}=-\frac{1}{r} \cot u^{1} e_{1}, & \nabla_{e_{0}} e_{1}=\frac{1}{r} \cot u^{1} e_{0}, \\
\nabla_{e_{2}} e_{1}=-\frac{1}{r} \tan u^{1} e_{2}, & \nabla_{e_{2}} e_{2}=\frac{1}{r} \tan u^{1} e_{1}
\end{array}
$$

and the remaining $\nabla_{e_{i}} e_{j}$ are zero.

Bearing in mind (2.2), (2.3) and (3.5), we obtain the following components $F_{i j k}$ of $F$ with respect to the basis $\left\{e_{i}\right\}, i \in\{0,1,2\}$ :

$$
F_{002}=F_{020}=\frac{1}{r} \cot u^{1}, \quad F_{211}=-F_{222}=\frac{2}{r} \tan u^{1}
$$

and the other components $F_{i j k}$ are zero.

According to (2.5), (2.6) and (3.6), we determine the basic components $N_{i j k}=$ $N\left(e_{i}, e_{j}, e_{k}\right)$ of the Nijenhuis tensor and $\widehat{N}_{i j k}=\widehat{N}\left(e_{i}, e_{j}, e_{k}\right)$ of its associated tensor. The non-zero of them are:

$$
\begin{aligned}
& N_{010}=-N_{100}=\frac{1}{r} \cot u^{1}, \\
& \widehat{N}_{221}=\widehat{N}_{111}=-\widehat{N}_{122}=-\widehat{N}_{212}=\frac{4}{r} \tan u^{1}, \\
& \widehat{N}_{001}=-\frac{2}{r} \cot u^{1}, \quad \widehat{N}_{010}=\widehat{N}_{100}=\frac{1}{r} \cot u^{1} .
\end{aligned}
$$

Using (2.4) and (3.6), we get the equality

$$
F(x, y, z)=\frac{2}{r} \tan u^{1} x^{2}\left(y^{1} z^{1}-y^{2} z^{2}\right)+\frac{1}{r} \cot u^{1} x^{0}\left(y^{0} z^{2}+y^{2} z^{0}\right) .
$$

By virtue of the latter equality, we establish that $F$ has the following form:

$$
F(x, y, z)=F^{1}(x, y, z)+F^{11}(x, y, z),
$$

where $F^{1}$ and $F^{11}$ are the components of $F$ for the basic classes $\mathcal{F}_{1}$ and $\mathcal{F}_{11}$, respectively. Therefore, we have the following non-zero components of $F^{1}$ and $F^{11}$ with respect to $\left\{e_{i}\right\}, i \in\{0,1,2\}$ :

$$
\begin{aligned}
& F_{211}^{1}=-F_{222}^{1}=-\theta_{2}=\frac{2}{r} \tan u^{1}, \\
& F_{002}^{11}=F_{020}^{11}=\omega_{2}=\frac{1}{r} \cot u^{1} .
\end{aligned}
$$


Let us note that the components of $F^{1}$ and $F^{11}$ from the above are non-zero for all values of $u^{1}$ in its domain.

Next, using (2.1), (3.7) and (3.8), we find the following:

$$
N=-\mathrm{d} \eta \otimes \xi, \quad \nabla_{\xi} \xi \neq 0,
$$

which support the obtained results in 7 .

Bearing in mind (2.3), (3.3), 3.5 and the definition equality of $R$, we obtain the components $R_{i j k \ell}=R\left(e_{i}, e_{j}, e_{k}, e_{\ell}\right)$ of $R$ with respect to $\left\{e_{i}\right\}, i \in\{0,1,2\}$. The first of them are:

$$
R_{0101}=R_{0202}=R_{1212}=-\frac{1}{r^{2}} .
$$

The rest of the non-zero components of $R$ are determined by (3.9) and the basic symmetries of $R$ and its first Bianchi identity.

According to (2.3), (2.7) and (3.9), we obtain the components $\rho_{j k}=\rho\left(e_{j}, e_{k}\right)$ and $\rho_{j k}^{*}=\rho^{*}\left(e_{j}, e_{k}\right)$ of the Ricci tensor $\rho$ and the $*$-Ricci tensor $\rho^{*}$, respectively, as well as the values of the scalar curvature $\tau$ and its associated quantity $\tau^{*}$ as follows:

$$
\begin{aligned}
& \rho_{00}=\rho_{11}=\rho_{22}=\frac{2}{r^{2}}, \quad \rho_{12}^{*}=\rho_{21}^{*}=-\frac{1}{r^{2}}, \\
& \tau=\frac{6}{r^{2}}, \quad \tau^{*}=0 .
\end{aligned}
$$

Futhermore, from (2.3), (2.8) and (3.9), we get the basic sectional curvatures $k_{i j}=$ $k\left(e_{i}, e_{j}\right)$ determined by the basis $\left\{e_{i}, e_{j}\right\}$ of the corresponding 2-plane:

$$
k_{01}=k_{02}=k_{12}=\frac{1}{r^{2}} \text {. }
$$

Taking into account (2.3), (3.9) and (3.11), we get the form of the curvature tensor as follows

$$
R(x, y, z, w)=-\frac{1}{2 r^{2}}(g \otimes g)(x, y, z, w) .
$$

According to the obtained results from the above, we have the following

Theorem 3.2. Let $\left(S_{1}, \phi, \xi, \eta, g\right)$ be the hypersphere in the Euclidean 4-space $\mathbb{E}^{4}$ equipped with an almost paracontact almost paracomplex structure and a Riemannian metric defined by (3.1), (2.2) and (2.3). Then, the manifold $\left(S_{1}, \phi, \xi, \eta, g\right)$ has the following properties:

(1) it is in the class $\mathcal{F}_{1} \oplus \mathcal{F}_{11}$ but does not belong to either $\mathcal{F}_{1}$ or $\mathcal{F}_{11}$;

(2) it has a positive scalar curvature;

(3) it is *-scalar flat;

(4) it is a space-form of positive constant sectional curvature.

Proof. We establish the truthfulness of assertion (1) using (3.7) and (3.8). Conclusions (2) and (3) are consequences of (3.10), whereas (4) follows from (3.12).

\section{A hypersphere with the studied structure in Minkowski 4-space}

In this section we consider the pseudo-Euclidean space $\mathbb{E}_{1}^{4}$, i.e. the real 4 -space $\mathbb{R}^{4}$ equipped with the following Lorentzian inner product

$$
\langle x, y\rangle=x^{1} y^{1}+x^{2} y^{2}+x^{3} y^{3}-x^{4} y^{4}
$$

for arbitrary vectors $x\left(x^{1}, x^{2}, x^{3}, x^{4}\right)$ and $y\left(y^{1}, y^{2}, y^{3}, y^{4}\right)$ in $\mathbb{R}^{4}$. 
In a similar manner as in the previous section, we define the following hypersphere $S_{2}$ in $\mathbb{E}_{1}^{4}$ at the origin with real radius $r$,

$$
S_{2}: \quad\langle z, z\rangle=-r^{2} .
$$

It has the following parametrization

$$
z\left(r \sinh u^{1} \cos u^{2}, r \sinh u^{1} \sin u^{2}, r \cosh u^{1} \sinh u^{3}, r \cosh u^{1} \cosh u^{3}\right),
$$

where $u^{1}, u^{2}, u^{3}$ are real parameters such as $u^{1} \in(-\infty ; 0) \cup(0 ;+\infty), u^{2} \in[0 ; 2 \pi)$, $u^{3} \in(-\infty ;+\infty)$.

Therefore, for the local basic vectors $\partial_{i}=\frac{\partial z}{\partial u^{i}}, i \in\{1,2,3\}$, we obtain the following

$$
\begin{aligned}
& \left\langle\partial_{1}, \partial_{1}\right\rangle=r^{2}, \quad\left\langle\partial_{2}, \partial_{2}\right\rangle=r^{2} \sinh ^{2} u^{1}, \quad\left\langle\partial_{3}, \partial_{3}\right\rangle=r^{2} \cosh ^{2} u^{1}, \\
& \left\langle\partial_{i}, \partial_{j}\right\rangle=0, i \neq j .
\end{aligned}
$$

Then, we substitute $e_{i-1}=\frac{1}{\sqrt{\left|\left\langle\partial_{i}, \partial_{i}\right\rangle\right|}} \partial_{i}$ and get the orthonormal basis $\left\{e_{i}\right\}$, $i \in\{0,1,2\}$, as follows

$$
e_{0}=\frac{1}{r} \partial_{1}, \quad e_{1}=\frac{1}{r \sinh u^{1}} \partial_{2}, \quad e_{2}=\frac{1}{r \cosh u^{1}} \partial_{3} .
$$

In the same way as in the previous section, here we equip $S_{2}$ with an almost paracontact almost paracomplex structure and a Riemannian metric defined by (2.2) and (2.3), respectively. Thus, for the obtained manifold $\left(S_{2}, \phi, \xi, \eta, g\right)$, we have the following

Proposition 4.1. The manifold $\left(S_{2}, \phi, \xi, \eta, g\right)$ is a 3-dimensional almost paracontact almost paracomplex Riemannian manifold.

By similar considerations as for $S_{1}$, we obtain the following:

$$
\left[e_{0}, e_{1}\right]=-\frac{1}{r} \operatorname{coth} u^{1} e_{1}, \quad\left[e_{0}, e_{2}\right]=-\frac{1}{r} \tanh u^{1} e_{2}, \quad\left[e_{1}, e_{2}\right]=0 .
$$

Taking into account (3.4) and (4.2) we get:

$$
\begin{array}{ll}
\nabla_{e_{1}} e_{0}=\frac{1}{r} \operatorname{coth} u^{1} e_{1}, & \nabla_{e_{2} e_{0}}=\frac{1}{r} \tanh u^{1} e_{2}, \\
\nabla_{e_{1}} e_{1}=-\frac{1}{r} \operatorname{coth} u^{1} e_{0}, & \nabla_{e_{2}} e_{2}=-\frac{1}{r} \tanh u^{1} e_{0} .
\end{array}
$$

Bearing in mind (2.2), (2.3) and (4.3), we compute the components $F_{i j k}$ of $F$. The non-zero of them are:

$$
F_{102}=F_{120}=-\frac{1}{r} \operatorname{coth} u^{1}, \quad F_{201}=F_{210}=-\frac{1}{r} \tanh u^{1} .
$$

Then, applying (2.5), (2.6) and (4.4), we calculate the components $N_{i j k}$ and $\widehat{N}_{i j k}$ as follows:

$$
\begin{aligned}
& N_{101}=-N_{011}=N_{022}=-N_{202}=\frac{2}{r \sinh 2 u^{1}}, \\
& \widehat{N}_{101}=\widehat{N}_{011}=-\widehat{N}_{202}=-\widehat{N}_{022}=\frac{2}{r \sinh 2 u^{1}}, \\
& \widehat{N}_{110}=\widehat{N}_{220}=-\frac{2}{r}\left(\operatorname{coth} u^{1}+\tanh u^{1}\right) .
\end{aligned}
$$

By vitue of (2.4) and (4.4), we establish the following equality

$$
F(x, y, z)=\left(F^{5}+F^{9}\right)(x, y, z),
$$


where $F^{5}$ and $F^{9}$ are the components of $F$ corresponding to the basic classes $\mathcal{F}_{5}$ and $\mathcal{F}_{9}$. The non-zero components of $F^{5}$ and $F^{9}$ with respect to the basis $\left\{e_{0}, e_{1}, e_{2}\right\}$ are the following

$$
\begin{aligned}
& F_{102}^{5}=F_{120}^{5}=F_{201}^{5}=F_{210}^{5}=\frac{1}{2} \theta_{0}^{*}=-\frac{1}{2 r}\left(\operatorname{coth} u^{1}+\tanh u^{1}\right), \\
& F_{102}^{9}=F_{120}^{9}=-F_{201}^{9}=-F_{210}^{9}=\mu=\frac{1}{2 r}\left(\tanh u^{1}-\operatorname{coth} u^{1}\right) .
\end{aligned}
$$

Taking into account (2.1), (4.5) and (4.6), we get

$$
\mathrm{d} \eta=0, \quad \nabla_{\xi} \xi=0,
$$

which support the obtained results in 7 .

Bearing in mind (2.3), (4.2) and (4.3), we calculate the components $R_{i j k l}$ of $R$. The non-zero of them are determined by the basic symmetries of $R$ and the following

$$
R_{0101}=R_{0202}=R_{1212}=\frac{1}{r^{2}} .
$$

Using (2.3), (2.7) and (4.8), we obtain the basic components $\rho_{j k}$ and $\rho_{j k}^{*}$ as well as the values of $\tau$ and $\tau^{*}$ :

$$
\begin{array}{ll}
\rho_{00}=\rho_{11}=\rho_{22}=-\frac{2}{r^{2}}, & \rho_{12}^{*}=\rho_{21}^{*}=\frac{1}{r^{2}}, \\
\tau=-\frac{6}{r^{2}}, & \tau^{*}=0 .
\end{array}
$$

Bearing in mind (2.3), (2.8) and (4.8), we compute the basic sectional curvatures $k_{i j}$ with respect to the basis $\left\{e_{0}, e_{1}, e_{2}\right\}$ as follows

$$
k_{01}=k_{02}=k_{12}=-\frac{1}{r^{2}} .
$$

Bearing in mind (2.3), (4.8) and (4.10), we get the form of the curvature tensor in the following way

$$
R(x, y, z, w)=\frac{1}{2 r^{2}}(g \otimes g)(x, y, z, w) .
$$

By virtue the obtained results from the above, we obtain the following

Theorem 4.2. Let $\left(S_{2}, \phi, \xi, \eta, g\right)$ be the time-like sphere in the Minkowski 4-space $\mathbb{E}_{1}^{4}$ equipped with an almost paracontact almost paracomplex structure and a Riemannian metric defined by (4.1), (2.2) and (2.3). Then, the manifold $\left(S_{2}, \phi, \xi, \eta, g\right)$ has the following properties:

(1) it is in the class $\mathcal{F}_{5} \oplus \mathcal{F}_{9}$ but does not belong to either $\mathcal{F}_{5}$ or $\mathcal{F}_{9}$;

(2) it has a closed 1-form $\eta$ and geodesic integral curves of $\xi$;

(3) it has a negative scalar curvature;

(4) it is $*$-scalar flat;

(5) it is a space-form of negative constant sectional curvature.

Proof. We establish the truthfulness of assertion (1) using (4.5) and (4.6). Statements (2), (3)-(4), (5) follow directly from (4.7), (4.9), (4.11), respectively.

Acknowledgment. The authors were supported by project of the Scientific Research Fund, University of Plovdiv Paisii Hilendarski, Bulgaria. 


\section{REFERENCES}

[1] T. Adati, T. Miyazawa: On paracontact Riemannian manifolds. TRU Math. 13 (1977), 27-39

[2] G. Ganchev, V. Mihova, K. Gribachev: Almost contact manifolds with B-metric. Math. Balkanica (N.S.) 7 (3-4) (1993), 261-276

[3] S. Kaneyuki, M. Konzai: Paracomplex structures and affine symmetric spaces. Tokyo J. Math. 8 (1985), 301-318

[4] P. Libermann: Sur les structures presque paracomplexes. C. R. Acad. Sci. I 234 (1952), $2517-2519$

[5] H. Manev: Space-like and time-like hyperspheres in real pseudo-Riemannian 4-spaces with almost contact B-metric structures. Novi Sad J. Math. 46 (2016), 181-189

[6] M. Manev, M. Staikova: On almost paracontact Riemannian manifolds of type $(n, n)$. J. Geom. 72 (2001), 108-114. https://doi.org/10.1007/s00022-001-8572-2

[7] M. Manev, V. Tavkova: On the almost paracontact almost paracomplex Riemannian manifolds. Facta Univ. Ser. Math. Inform. 33 (2018), 637-657

[8] M. Manev, V. Tavkova: Lie groups as 3-dimensional almost paracontact almost paracomplex Riemannian manifolds. J. Geom. 110 (2019), 43. https://doi.org/10.1007/s00022-019-0499-6

[9] M. Manev, V. Tavkova: Matrix Lie groups as 3-dimensional almost paracontact almost paracomplex Riemannian manifolds. arXiv:2005.07061

[10] A.M. Naveira: A classification of Riemannian almost product manifolds. Rend. Mat. (7) 3 (1983), 577-592

[11] S. Sasaki: On paracontact Riemannian manifolds. TRU Math. 16 (1980), 75-86

[12] I. Satō: On a structure similar to the almost contact structure. Tensor (N.S.) 30 (1976), $219-224$

[13] S. Zamkovoy, G. Nakova: The decomposition of almost paracontact metric manifolds in eleven classes revisited. J. Geom. 109 (2018), 18. https://doi.org/10.1007/s00022-018-0423-5

(MM1, VT) Department of Algebra and Geometry, Faculty of Mathematics and Informatics, University of Plovdiv Paisit Hilendarski, 24, Tzar Asen St, 4000 Plovdiv, BULGARIA

Email address: mmanev@uni-plovdiv.bg

Email address: vtavkova@uni-plovdiv.bg

(MM2) Department of Medical Informatics, Biostatistics and E-Learning, Faculty of Public Health, Medical University of Plovdiv, 15A, Vasil Aprilov Blvd, 4002 Plovdiv, BULGaRia 